\title{
CARCINOME HYBRIDE DE LA FOSSE NASALE
}

\author{
R. BEN M'HAMED, S. MEZRI, C. ZGOLLI I, N. MATHLOUTHI, H. BRAHEM, M. BALTI, \\ K. AKKARI, S. BENZARTI, M K. CHEBBI \\ SERVICE D'ORL ET DE CHIRURGIE MAXILLO-FACIALE \\ HOPITAL MILITAIRE DE TUNIS
}

\begin{abstract}
Les carcinomes hybrides sont des tumeurs rares représentant moins de $0,1 \%$ des tumeurs des glandes salivaires. Un seul cas de carcinome hybride nasosinusien a été rapporté à ce jour.

Nous rapportons le cas d'une patiente âgée de 56 ans présentant une tumeur à double contingent carcinomateux épithélial-myoépithélial et adénoïde kystique développée au niveau de la fosse nasale droite, dont l'exérèse par voie de Caldwell-Luc s'est soldée par une récidive au terme de deux ans d'évolution. Une deuxième chirurgie a été réalisée par voie de Rouge Denker avec une exérèse emportant la récidive localisée mais respectant les structures ostéocartilagineuses des fosses nasales, complétée d'une radiothérapie du lit tumoral et des aires ganglionnaires cervicales. Nous ne disposant pas d'assez de recul pour parler de l'évolution. II s'agit du deuxième cas de tumeur hybride à localisation nasale rapporté à ce jour.

Les tumeurs hybrides carcinomateuses à localisation naso-sinusienne sont possibles bien qu'exceptionnelles. Leur prise en charge thérapeutique et leur pronostic sont corolaires du contingent le plus agressif.
\end{abstract}

Mots-clé : Carcinome; hybride; tumeurs fosses nasales.

Hybrid carcinoma is a rare neoplasm, accounting for less than $0.1 \%$ of all registered tumors in salivary glands. Up to now, only one case of hybrid carcinoma of the nasal cavity has been described.

In this report, we describe a case of hybrid carcinoma composed of epithelial-myoepithelial carcinoma with an adenoid cystic carcinoma component occurring in the nasal cavity in a 56-year-old woman. Caldwell-Luc means resection was performed and the patient revisited because of the tumor recurrence within two years of evolution. A second surgery was accomplished by means of Red Denker and a adjuvant radiation therapy is envisaged. We have not enough detachment to speak about actual evolution. It is in our knowledge the second case of hybrid tumor of the nasal cavity described in the literature.

Hybrid carcinoma of the nasal cavity is possible although rare. Therefore recognition of other component that is more aggressive and has a worse prognosis has therapeutic and prognostic ramifications.

Keywords: Carcinoma; hybrid; tumors of nasal cavity.

\section{INTRODUCTION}

De multiples tumeurs sont possibles au niveau des glandes salivaires. La tumeur hybride est une tumeur unique prenant naissance dans un même site anatomique et composée de deux contingents. Le nombre limité de cas rapportés par la littérature fait qu'il est difficile de proposer une conduite thérapeutique et d'établir un pronostic. Nous rapportons un cas de tumeur hybride à localisation nasale chez une femme de 56 ans avec un recul de deux ans.

\section{OBSERVATION}

Mme L.Z âgée de 56 ans, diabétique insulinodépendante, s'est présentée à notre consultation en Octobre 2006 pour une obstruction nasale droite associée à une épistaxis récidivante homolatérale, le tout évoluant depuis huit mois. Le bilan endoscopique a objectivé une formation polypoïde à surface lisse tapissé par un fin réseau capillaire occupant la partie postérieure de la fosse nasale droite arrivant au contact du plancher nasal et atteignant la choane. Le reste de l'examen somatique était normal. L'examen tomodensitométrique (Fig.1, 2) du massif facial a objectivé une formation tissulaire occupant la choane droite, isodense par rapport aux muscles se rehaussant modérément après injection de produit de contraste. Cette masse ne présente pas d'extension au rhinopharynx, le sinus maxillaire droit était comblé mais aucune lyse osseuse n'a été objectivée. L'IRM n'a pu être réalisée car la patiente est claustrophobe. Une exérèse par voie de Caldwell-Luc a été réalisée. L'examen anatomopathologique de la pièce opératoire avec étude immunohistochimique a conclu à une tumeur hybride associant un double contingent carcinomateux épithélial- 
myoépithélial et adénoïde kystique sans embols vasculaires ni engainement péri-nerveux, les limites de résection étaient saines. Il a été décidé de sursoir à une radiothérapie postopératoire. La patiente a été perdue de vue pendant deux ans, au terme desquels elle reconsulte pour la même symptomatologie avec une récidive locale isolée toujours sans extension locorégionale (Fig. 3). Le bilan d'extension à distance comportant une radiographie du thorax, une échographie abdominale et une scintigraphie osseuse étaient sans anomalies. La patiente a été reprise par voie de Rouge Denker avec exérèse de la récidive localisée tout en respectant les structures ostéocartilagineuses des fosses nasales. Les suites postopératoires étaient simples. La patiente a reçu un complément thérapeutique par radiothérapie externe (60 Gy étalés sur 30 séances sur le lit opératoire des fosses nasales et le 1er relai ganglionnaire et 50 Gy sur le reste des ganglions cervicaux). Après un recul de trois mois, on ne note pas de récidive.

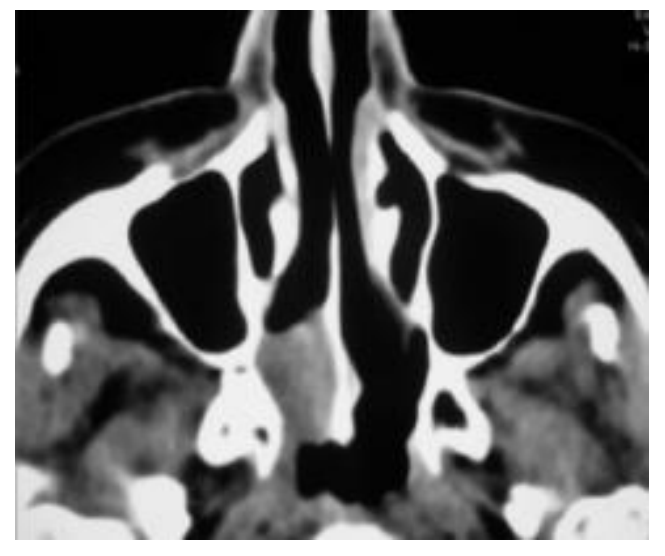

Fig. 1 : TDM coupe axiale objectivant la présence au niveau de la partie postérieure de la fosse nasale droite d'une formation tissulaire iso dense par rapport aux muscles.

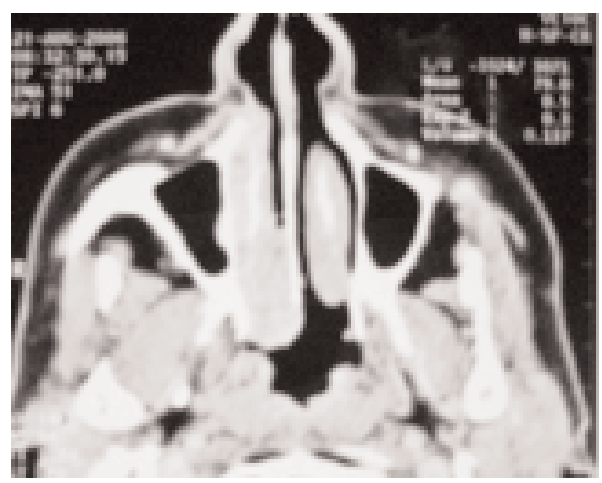

Fig. 2 : TDM coupe axiale montrant la même masse tissulaire de la fosse nasale droite se réhaussant modérément à l'injection de PDC. Absence de lyse osseuse.

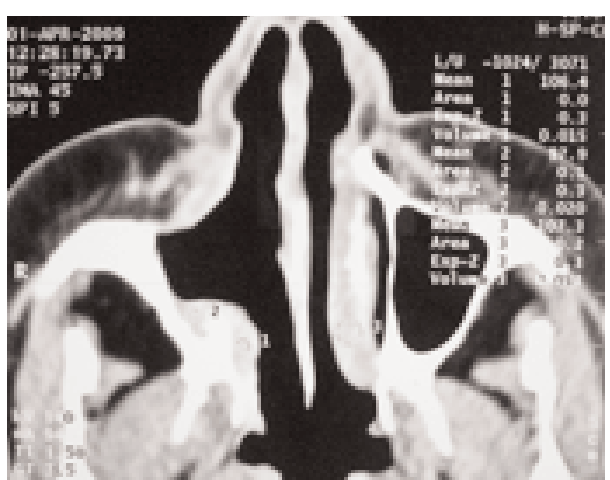

Fig. 3 : TDM coupe axiale montrant un épaississement tissulaire de la paroi intersinusienne

\section{DISCUSSION}

Les tumeurs hybrides sont définies par l'association de deux tumeurs différentes se développant dans le même site anatomique et donnant naissance à une masse tumorale unique aussi bien macroscopiquement que microscopiquement $(1,2)$. C'est une forme rare des tumeurs des glandes salivaires dont la fréquence est inférieure à $0,1 \%$ de toutes les tumeurs des glandes salivaires (1).

Les rares cas de tumeurs hybrides rapportés dans la littérature intéressent principalement la glande parotide suivie par la glande submandibulaire et le palais. A notre connaissance, un seul cas de carcinome hybride à localisation maxillaire a été rapporté à ce jour par une équipe coréenne (3).

La composante épithéliale-myoépithéliale de ce carcinome est plus fréquente chez la femme avec une fréquence de $60 \%(4,5)$, et un âge moyen de découverte à 60 ans avec des extrêmes allant de 08 à 103 ans (4,5). Quant au contingent carcinomateux adénoïde kystique, il intéresse essentiellement les glandes salivaires accessoires surtout celles localisées au niveau de la cavité buccale. La localisation nasosinusienne a été rarement rapportée (6).

Le diagnostic de cette tumeur à progression lente est généralement porté plusieurs mois, voire plusieurs années après l'apparition des premiers symptômes.

Les signes fonctionnels amenant le malade à consulter n'ont rien de spécifique; il s'agit fréquemment d'obstruction nasale unilatérale pouvant devenir bilatérale, d'épistaxis récidivantes ou d'algie faciale.

L'aspect endoscopique de cette tumeur est celui d'une lésion polypoïde ulcérée par endroit saignant au contact pouvant atteindre plusieurs centimètres de diamètre prenant naissance au niveau de la paroi intersinusonasale en regard du méat moyen.

Le bilan radiologique comporte au mieux une tomodensitométrie complétée par une imagerie par résonnance magnétique des sinus de la face qui vont préciser l'extension locorégionale et à la base du crâne ainsi que l'extension péri-nerveuse apanage de la composante adénoïde kystique de ces carcinomes hybrides.

Le diagnostic anatomopathologique est étayé par une étude immunohistochimique éventuellement complétée 
par la microscopie électronique.

Le contingent carcinomateux le plus agressif est important à préciser, car c'est lui qui va conditionner la prise en charge thérapeutique et le pronostic. Quant au carcinome épithélial-myoépithélial, il est classé parmi les tumeurs de bas grade de malignité $(7,8,9)$ et son caractère malin est mis en doute par certaines équipes $(8,9)$.

Le nombre limité de cas rapportés rend la standardisation d'une prise en charge thérapeutique impossible. Cependant, toute les équipes s'accordent à dire qu'une exérèse chirurgicale complète en monobloc est primordiale afin d'éviter les récidives $(10,11)$. La radiothérapie postopératoire améliore le pronostic à long terme des patients ayant de volumineuses masses tumorales, en diminuant la fréquence des récidives locales $(10,11)$. Les principales indications sont : l'existance d'une composante carcinomateuse, une taille tumorale dépassant les quatre centimètres et des limites de résection envahies $(10,11,12)$. La neutronthérapie rapportée par certains donnerait de meilleurs résultats que l'irradiation classique (13). L'effet de la chimiothérapie n'est pas démontré.

De part son évolution lente, le carcinome épithélialmyoépithélial donne plus de récidives locales, pouvant atteindre selon les séries $50 \%$ des cas $(7,14)$ et des métastases à distance dans une proportion allant de 8 à $25 \%$ des cas (7). Le bilan d'extension doit donc comporter systématiquement une radiographie du thorax, une échographie abdominale et une scintigraphie osseuse. Le pronostic du carcinome adénoïde kystique, tumeur plus agressive, est grevé d'une lourde mortalité à court terme (10). Notre patiente est toujours en vie au terme de deux ans d'évolution malgré l'absence d'irradiation initiale.

Le potentiel évolutif de ces tumeurs rend donc une surveillance clinique et radiologique rapprochée et prolongée obligatoire.

\section{CONCLUSION}

L'association d'un carcinome épithélial-myoépithélial à un carcinome adénoïde kystique au niveau de la cavité nasale est exceptionnelle. Sa rareté explique la difficulté d'établir une prise en charge thérapeutique codifiée, cependant, il faut tenir compte du contingent ayant le plus mauvais pronostic imposant une surveillance régulière et prolongée.

\section{REFERENCES}

1. Seifert G, Donath K. Hybrid tumours of salivary glands: definition and classification of five rare cases. Eur J Cancer B 1996; 32B: 251-9.

2. Seifert G, Donath K. Multiple tumours of the salivary glands: terminology and nomenclature. Eur J Cancer Oral Oncol 1996; 32B: 3-7.

3 .Jeong-Su Woo, Soon-Young Kwon, Kwang-Yoon Jung, Insun Kim : A Hybrid Carcinoma of Epithelial-Myoepithelial carcinoma and Adenoid Cystic Carcinoma in Maxillary Sinus : J Korean Med Sci $2004 ; 19: 462-5$.

4. Hamper K, Brugman M, Koppormann R, et al. Epithelial-myoepithelial carcinoma of the salivary glands: a follow up cytophotometric study of 21 cases. J Oral Pathol Med 1989; 18: 299-304.

5. Fonseca I, Felix A, Soares J: Cell proliferation in salivary gland adeno-carcinoma with myoepithelial participation. A study of 78 cases. Wirchow Arch 1997: 430: 277-82.

6. Bradley PJ. Adenoid cystic carcinoma of the head and neck: a review. Curr Opin Otolaryngol Head Neck Surg.2004: 12: 27-32.

7. Fonseca J, Soares J. Epithelial-myoepithelial carcinoma of the salivary glands: a study of 22 cases. Virchow Arch A Path Anat Histopathol 1993; 422: 89-96.

8. Kasper HU, Mellin W, Cheremet E, Roessner A. Epithelial-myoepithelial carcinoma: a low grade malignant neoplasm?Report of two cases and review of literature. Pathol Res Pract 1999; 195: 189-92.
9. Friederich RE, Donath K. Epithelial-myoepithelial carcinoma of the parotid gland with multiple metastases: a case report. J Oral Maxillofac Surg 2000; 58: 690-4.

10. Konno A, Ishikawa K, Numata T, Negata H, Terada N, Okamato Y, Analysis of factors affecting long-term treatment results of adenoid cystic carcinoma of the nose and paranasal sinuses. Acta otolaryngol. 1998; 537: 67-74

11. Witterik IJ, Noyek AM, Chapnik JS, Hearthcote JG, Bedard YC. Observation of the natural history of a parotid epithelial myoepithelial carcinoma of intercalated ducts. J Otolaryngol 1993; 22: 176-79.

12. Kasper HU, Roessner A. Epithelial-myoepithelial carcinoma. Am J Surg Pathol 2000; 24: 308-9.

13. Prott F-J, Micke O, Haverkump U, Potter R. Results of fast neutron therapy of adenoid cystic carcinoma of the salivary glands. Anticancer Res. 2000; 20: 3743-50.

14. Micco AG, Shattuck MC, Wolff AP. Pathologic quiz 2; Arch Otolaryngol Head and Neck Surg 1991; 117: 926-9. 